\section{TEGAVIVINT REDUCES THE IMMUNOSUPPRESSIVE MACROPHAGE PHENOTYPE IN A PRECLINICAL CO- CULTURE MODEL OF THE NON-SMALL CELL LUNG CANCER TUMOR MICROENVIRONMENT}

${ }^{1}$ Raghav Chandra*, 'Josiah Flaming, ${ }^{1}$ Aiden Nguyen, 'Michael Peyton, ${ }^{1}$ Amit Das, ${ }^{1}$ Boning Gao, ${ }^{2}$ Steven Horrigan, ${ }^{1}$ Rolf Brekken, ${ }^{1} J o h n$ Minna. 'University of Texas Southwestern Medical Center, Dallas, TX, USA; ${ }^{2}$ Iterion Therapeutics, Houston, TX, USA

Background Activation of the Wnt/B-catenin pathway in nonsmall cell lung cancer (NSCLC) is associated with tumor growth and metastasis. Activation of this pathway in tumor cells may also modulate the immune tumor microenvironment (TME) by polarizing macrophages into an immunosuppressive M2-like phenotype. We tested whether treatment of co-cultures of human NSCLC cells, cancer-associated fibroblasts (CAFs) and macrophages with Tegavivint (Iterion Therapeutics), a novel compound that targets TBL1 to inhibit ß-catenin function that is in early-phase clinical trials, altered the phenotype of mouse macrophages in vitro.

Methods Our prior work testing a large panel of patientderived NSCLC lines in co-cultures of CAFs and mouse bone marrow-derived macrophages (BMDMs) demonstrated that individual NSCLC lines reproducibly generated several macrophage phenotypes, one of which was immunosuppressive (high expression of Arginase-1). We prepared co-cultures of NSCLC cells (H1666 and H2009) (70\%), human CAFs (25\%) and mouse BMDMs (5\%) and demonstrated conversion to the macrophage high Arginase-1 immunosuppressive phenotype by monitoring quantitative expression of mouse genes with qRTPCR. Expression of $\beta$-catenin protein relative to GAPDH by Western blot was determined before and after treatment with Tegavivint (10 and $20 \mathrm{nM})$. Co-cultures were treated with Tegavivint $(10 \mathrm{nM})$ for 48 and 72 hours and qRT-PCR for mouse macrophage-specific genes reflecting an immunosuppressive M2-like or an inflammatory, immunostimulatory M1-like phenotype (Arginase-1 and iNos, respectively) was performed. Positive controls consisted of stimulation of macrophages with IL-4 and LPS (to generate M2-like and M1-like phenotypes, respectively). Cytotoxicity assays (MTS and crystal violet) to determine the IC50 of Tegavivint were performed on NSCLC and macrophage cells.

Results Tegavivint IC50 values for NSCLCs were $17-40 \mathrm{nM}$. At $10 \mathrm{nM}$, Tegavivint had no cytotoxic effect on macrophages. Treatment with Tegavivint decreased NSCLC expression of B-catenin by $\sim 30-40 \%$. When co-cultures of NSCLCs, human CAFs, and mouse BMDMs were treated with $10 \mathrm{nM}$ Tegavivint, we found that macrophage expression of Arginase1 was significantly inhibited at 48 and 72 hours of treatment (figure 1).

Conclusions Tegavivint, at pharmacologically achievable doses and concentrations that have little or no cytotoxic effect on NSCLCs or macrophages, decreases tumor cell ß-catenin expression, and reduces the immunosuppressive macrophage phenotype (reduced macrophage Arginase-1 expression) induced by co-cultures of patient-derived NSCLCs and CAFs. These preclinical data set the stage for future clinical translation of Tegavivint as a NSCLC therapeutic in combination with immunotherapy approaches.

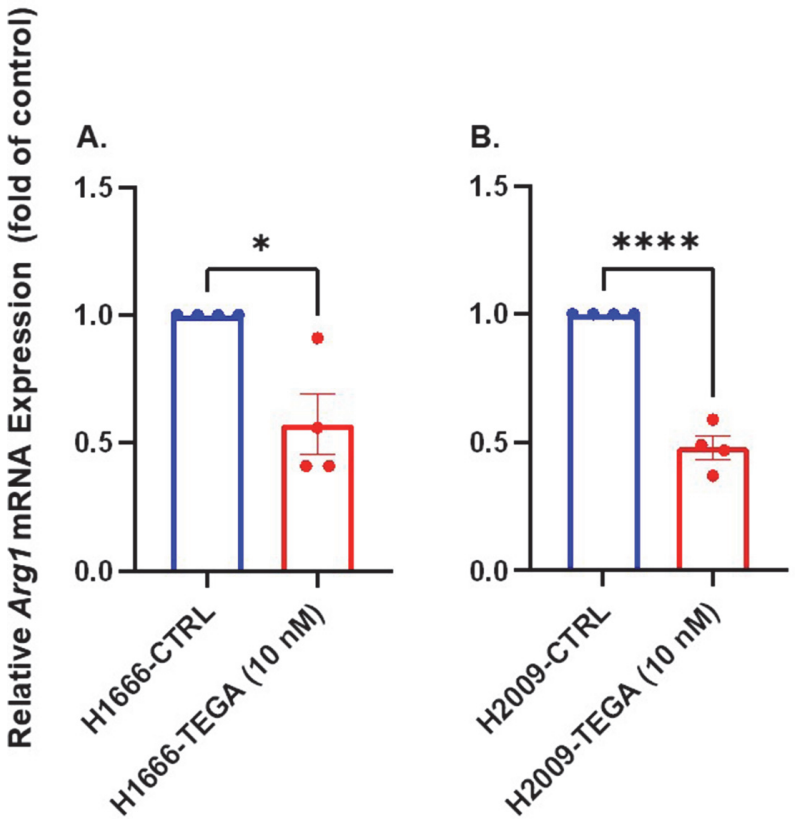

Abstract 919 Figure 1

Tegavivint reduces Arginase-1 mRNA expression from macrophages co-cultured with CAFs and H1666 (A) and H2009 (B) patient-derived NSCLC cell lines after 72-hour treatment. ${ }^{*}: p<0.05,{ }^{* * *}: p<0.0001$.

Acknowledgements Supported by P50 CA070907 and Physician-Scientist Institutional Award from the Burroughs Wellcome Fund (TARDIS Fellowship)

http://dx.doi.org/10.1136/jitc-2021-SITC2021.919 